Original article

\title{
A rat model for inducing temporomandibular anterior disc displacement experimentally
}

\author{
Ngan G. K. Nguyen, Akira Nishiyama, and Masahiko Shimada \\ Orofacial Pain Management, Oral Health Sciences, Graduate School of Medical and Dental Sciences, Tokyo Medical and Dental University, Tokyo, Japan
}

(Received March 2, 2019; Accepted May 13, 2019)

\begin{abstract}
The aim of this study was to establish an experimental rat model of temporomandibular joint (TMJ) anterior disc displacement (ADD). A pilot study was conducted to determine the most appropriate surgical protocol. In the main experiment, 40 rats were used. Twenty-four rats were subjected to ADD in the right TMJ, and subsequently thereafter six, nine, and nine rats were sacrificed at 1,4 , and 8 weeks, respectively, for gross evaluation. Twelve rats that underwent a sham operation were equally divided and sacrificed at each of the above time points. Four nontreated control rats were sacrificed at the beginning of the study. TMJ blocks were harvested for radiological and histological assessment. Gross examination showed that 14 rats in the ADD group (58.3\%) had anterior displacement of the TMJ disc. In the ADD joints, posterior condylar cartilage thickness decreased during the follow-up period; however, there was no significant difference between the sham-treated and ADD joints, or among the follow-up time points $(P>0.05)$. The anterior condylar cartilage exhibited obvious qualitative alterations. Radiologic signs of osteoarthrosis appeared after ADD surgery, but this became attenuated with time. The model investigated in this study successfully induced ADD in rats, and should be useful for assessment of progressive changes in the TMJ following ADD.
\end{abstract}

Keywords; condylar cartilage, fibrosis, posterior attachment, remodeling, temporomandibular disc

\section{Introduction}

Temporomandibular disorder (TMD) encompasses a group of musculoskeletal and neuromuscular conditions that involve the temporomandibular joint (TMJ), the masticatory muscles, and all associated tissues [1]. The most common form of intra-articular derangement in the TMJ is anterior disc displacement (ADD), which has a high incidence, occurring in $78-86.2 \%$ of TMD patients and $34-35 \%$ of asymptomatic individuals $[2,3]$. A systematic review has reported that its prevalence ranges from $18 \%$ to $35 \%$ in the general population [4]. Despite an extensive range of accessible literature, the role of ADD in TMD etiology remains controversial. It has been suggested that ADD can induce pain, masticatory dysfunction, and joint degeneration, and thus may be a progressive disorder; however, many researchers consider ADD to be a relatively stable and painless condition of the TMJ [4,5].

Various approaches have been used to investigate changes in the TMJ associated with $\mathrm{ADD}$, including conventional X-ray, computed tomography $(\mathrm{CT})$, and magnetic resonance imaging (MRI). Additionally, finite element models of the TMJ have been used to assess the etiological factors of TMD, focusing on overloading forces and stress analysis [6-8]. However, these methods make it difficult to explore details of pathogenesis in the living body. The TMJ in cadavers is useful for histological evaluation, but for decedents lacking a clear dental medical history, the results of inspection are difficult to interpret $[9,10]$. Surgical specimens collected from patients can also aid in understanding disease progression; however, such materials are limited [11-13]. Therefore, it is necessary to create an

Correspondence to Dr. Akira Nishiyama, Orofacial Pain Management, Oral Health Sciences, Graduate School of Medical and Dental Sciences, Tokyo Medical and Dental University, 1-5-45 Yushima, Bunkyo-ku, Tokyo 113-8549, Japan

Fax:+81-3-5803-5713 E-mail: anishi.tmj@tmd.ac.jp

Color figures can be viewed in the online issue at J-STAGE.

doi.org/10.2334/josnusd.19-0093

DN/JST.JSTAGE/josnusd/19-0093 animal model of ADD to evaluate its effect on TMJ structures.

Because of their structural and functional similarity to humans, rabbits and rats have been widely used for studying the TMJ [14-16]. Many surgery-induced ADD models have been created in rabbits [17-22]. Initially, these models were created by opening the TMJ capsule, removing the discal attachment, and pulling the disc anteriorly $[17,18]$. In some later studies, the capsule was opened and a traction suture was placed in the joint space while retaining the attachment of the disc $[19,20]$. More recent studies have induced ADD without the need to open the TMJ capsule $[21,22]$. Rats have also been used to study the TMJ and its functional and/ or pathological changes, such as those involving the TMD. Some studies have observed changes in the TMJ following articular discectomy [23-25]. In addition, rat models have been used to evaluate the effects of various factors contributing to TMD, such as mechanical stress, female hormones, and psychological factors [26,27].

Cinephotography and cinefluorography studies have demonstrated that rabbits and rats share many identical features of mandibular movement, including elevation and depression, protrusion and retraction, or movement from side to side $[28,29]$. During masticatory movements, despite the lack of a clear articular eminence, the condyle of the rat retains rotation and translation in two functional positions: the anterior part of the fossa and the posterior facet on the fossa $[29,30]$. For this reason, rats are a suitable alternative to rabbits for studies of intra-articular derangement of the TMJ. No previously reported study has assessed ADD in rats. Therefore, the aim of the present study was to investigate structural changes in the TMJ using an experimental rat model of ADD and to evaluate its feasibility as an alternative animal model of TMJ ADD involving surgical intervention.

\section{Materials and Methods}

\section{Study design}

First, a pilot study using eight rats was conducted to familiarize the investigators with rat anatomy (TMJ structures and surrounding tissues). Through this preliminary experiment, the surgical protocol for the main study was also established.

In the main experiment, 40 16-week-old male Wistar rats weighing between 450 and $515 \mathrm{~g}$ were used. Rats of this age are considered as adults $[31,32]$. Four rats serving as normal controls were sacrificed at the beginning of the experiment, and the remaining 36 were randomized into two surgical groups: sham group $(n=12)$ and experimental anterior disc displacement (ADD) group $(n=24)$. In the surgical groups, the right TMJ was chosen for experimental manipulation and the left TMJ was left untreated as a control. In the sham group, four animals were randomly sacrificed at 1 , 4 , and 8 weeks after surgery. In the ADD group, six, nine and nine rats were randomized for sacrifice at 1,4 , and 8 weeks after surgical manipulation, respectively.

The experimental protocols were approved by the Institutional Animal Experiment Committee of Tokyo Medical and Dental University, Japan (approval number: A2017-248A and A2018-027A), and all procedures were conducted according to the Guidelines for Animal Care and Use Standards.

\section{Surgical protocols}

Each experimental rat was anesthetized using 4\% Isoflurane, followed by general anesthesia with an intraperitoneal injection of Medetomidine hydrochloride $(0.375 \mathrm{mg} / \mathrm{kg}$ ), Midazolam (2 $\mathrm{mg} / \mathrm{kg}$ ), and Butorphanol $(2.5 \mathrm{mg} / \mathrm{kg})$. The right preauricular region was shaved and sterilized with iodine and alcohol. The surgical region was locally anesthetized with $1 \mathrm{~mL}$ 

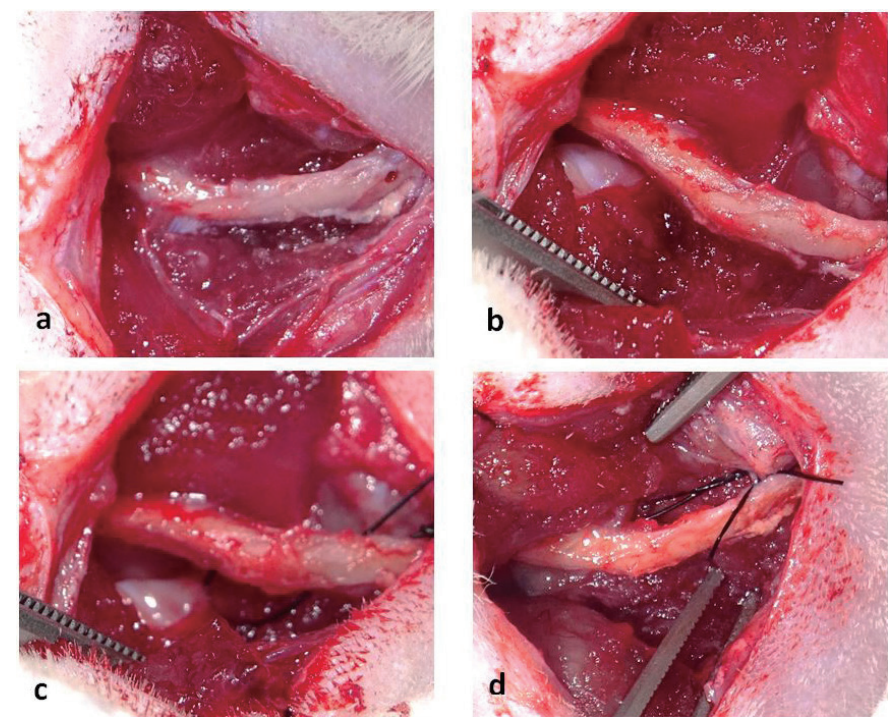

Fig. 1 Surgical process for creating ADD. a, drill a hole in the zygomatic arch; b, identify the disc c, penetrate the anterior part of the disc with suture; $d$, fix the suture to the prepared hole

Lidocaine (2\%). An incision approximately $2 \mathrm{~cm}$ long was made along the zygomatic arch, just anterior to the external auditory meatus and extending inferiorly to the lateral canthus of the eye. The overlying soft tissue was reflected to expose the zygomatic arch, revealing two junctions on the bone. A hole was drilled $1 \mathrm{~mm}$ anterior to the front junction using a round bur $(\varphi=0.5 \mathrm{~mm})$ with a low-speed handpiece under continuous saline irrigation. Thereafter, the lateral joint capsule was exposed, and the superior joint space was entered by splitting the synovial membrane attachment with micro-spring scissors. The disc and posterior attachment (PA) were identified. All discal attachments were left intact. The disc was kept in an anterior position, and the anterior part of the disc was penetrated by a round needle attached to 4/0 nylon suture (Natsume Seisakusho Co., Ltd., Tokyo, Japan). After pulling the disc forward, the other end of the suture was fixed to the prepared hole in the zygomatic arch (Figs. 1, 2). Care was taken to avoid injury to the articular soft tissue, especially the condylar cartilage surface. After displacement, the wound was thoroughly irrigated with saline, and the anterior position of the disc was verified. The surgical procedure was considered successful when the PA remained above the condyle in the closed mouth position. Finally, the wound was washed and closed in layers.

Rats in the sham group underwent the same treatments including zygomatic exposure and superior joint space access; however, the disc was merely mobilized without pulling it forward.

The surgery was performed using the SurgiSuite Multi-Function Surgical Platform (Kent Scientific Corporation, Torrington, CT, USA). During the operation, the rats were kept warm with an electrical heating pad placed on top of the platform. The surgical field was magnified $9 \times$ via a clip-on magnifier and head-mounted binocular loupes.

Postoperatively, antibiotics were injected intramuscularly daily for 3 days. The animals were fed food pellets and water ad libitum. A soft diet was also provided for the first week. All animals survived throughout the follow-up period of the study.

\section{Gross examination and specimen preparation}

At the sacrifice time-points, all animals were euthanatized by $\mathrm{CO}_{2}$ inhalation. For the ADD groups, gross examination was performed to check the status of the displacement. After careful dissection down to the superior joint cavity, cases were considered successful if the posterior attachment was above the condyle in the jaw-closed position.

The heads of successful ADD, sham, and non-surgically treated rats were dissected, skinned, and fixed in $10 \%$ neutral-buffered formalin $(\mathrm{pH}$ 7.4) for 24-48 h. The upper and lower jaws were maintained in the intercuspal position during the fixation process. Next, TMJ blocks approximately 2 $\times 2 \times 2 \mathrm{~cm}$ in size were harvested bilaterally, ready for micro-CT scanning and histological analysis.
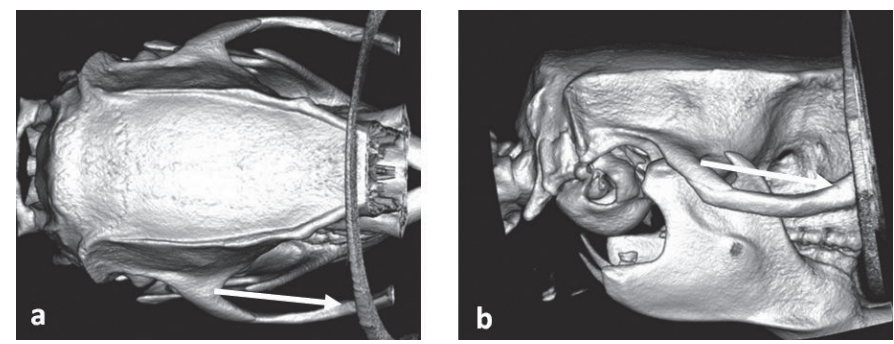

Fig. 2 Three-dimensional reconstruction images of the rat head. a, superior (top) view; b, lateral view; Arrow, position of nylon suture used to pull the articular disc anteriorly

\section{Micro-CT scanning}

The TMJ blocks were scanned using a micro-CT system, R-mCT2 (CosmoScan GX, Rigaku Corporation, Tokyo, Japan). The following imaging conditions were set for the present study: tube voltage, $90 \mathrm{kV}$; tube current, $160 \mu \mathrm{A}$ : voxel size, $20 \times 20 \times 20 \mu \mathrm{m}$; exposure time, $3 \mathrm{~min}$. Reconstruction time was approximately $20 \mathrm{~s}$. The slices were scanned parallel to the condylar lateromedial plane and 512 frames were collected. The reconstructed images were processed by I-View-R software (Rigaku Corporation). A $\times 2$ magnifying glass was used for the radiographic readings. Osseous abnormalities of the condyle were evaluated as cortical erosion, concavity, flattening, and osteophyte. Condylar cortical bone was considered normal if none of these signs were detected [33].

\section{Histological examination}

After taking micro-CT images, all specimen blocks were decalcified for 5 days, then dehydrated in an ascending graded alcohol series for 3 days followed by chloroform and subsequently embedded in paraffin.

The paraffin-embedded specimens were cut sagittally throughout the lateral to the medial part of the TMJ using a manual diamond saw. Sections $5 \mu \mathrm{m}$ thick were cut and collected on coated New Silane III micro slides (Muto Pure Chemicals Co., Ltd., Tokyo, Japan). The slides were stained with hematoxylin and eosin (HE) for qualitative and quantitative assessment. Three continuous sagittal sections at the central part were chosen, and images were captured at $\times 100$ magnification using a light microscope (Olympus BX41, Tokyo, Japan). The condylar cartilage thickness was measured at the posterior region in these three sections using Image-J software (National Institute of Health, Bethesda, MD, USA), then averaged [34]. Qualitative analysis was performed at the anterior and posterior region of the condylar cartilage.

\section{Statistical analysis}

Results are presented as the mean \pm standard deviation (SD). The Shapiro-Wilk test was used to confirm the normal distribution of the data. Nonparametric tests were applied in the case of nonparametric data. Body weight data were compared using Student's $t$-test between the sham and ADD groups. For comparison of posterior condylar cartilage thickness between two surgical groups and among follow-up time points, the Mann-Whitney $U$ test and the Friedman test were applied, respectively. If significance was recognized, post-hoc test was performed using the Wilcoxon sign-rank test with Bonferroni adjustment. Differences were considered significant at $P<0.05$. SPSS Ver.23 (IBM, Tokyo, Japan) was used for all statistical analyses.

\section{Results}

\section{Results of the pilot study}

In the pilot study, two surgical protocols were used: 1, severing the discal (lateral, medial, and anterior) attachments; and 2, inducing ADD while preserving these attachments. Rats treated using technique 1 showed severe changes of the condylar cartilage (histological assessment) and morphologic condylar bone (seen on micro-CT scan), which might have resulted from the aggressive surgical approach rather than displacement of the disc. Moreover, technique 2 enabled anterior dislocation of the disc to be performed. Therefore, in the surgical protocol for the main study, the discal attachments were retained. 


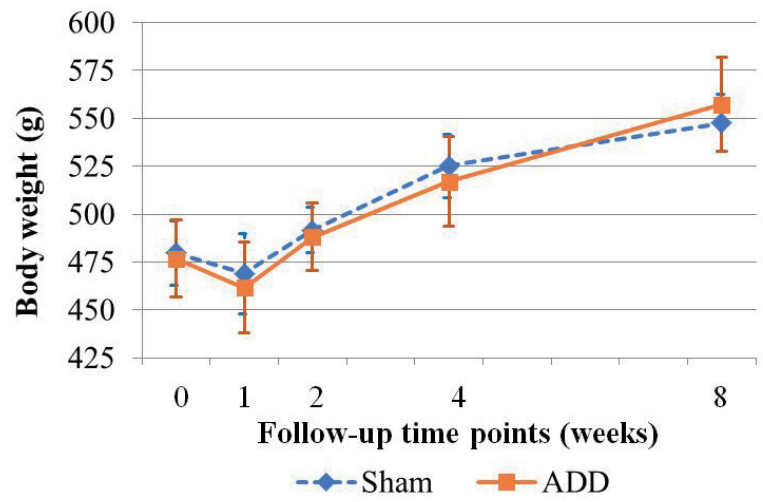

Fig. 3 Body weight characteristics. Data are shown as the mean \pm SD. Numbers of rats in the sham group were $12,12,8,8$, and 4 , and those in the ADD group were $24,24,18,18$, and 9 at $0,1,2,4$, and 8 weeks, respectively. $\mathbf{a}$

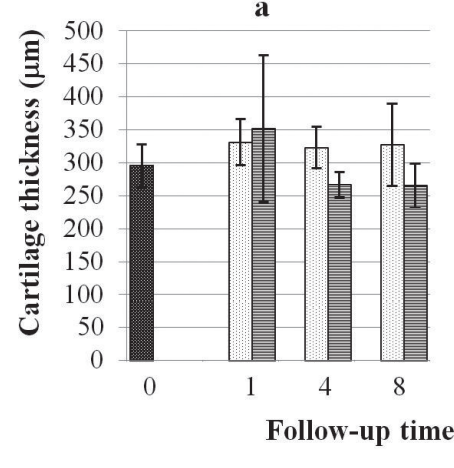

b

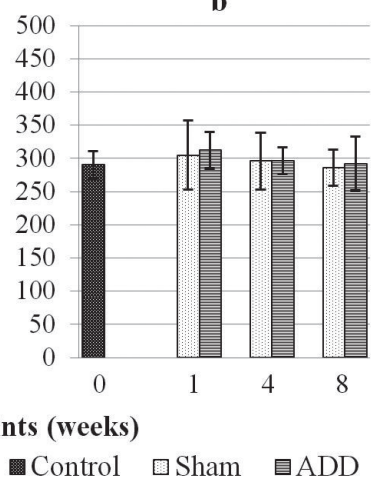

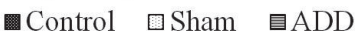
SD. Control: non-treated rats $(n=4)$; Sham: rats given a sham operation $(n=4$ at each follow-up time-point); ADD: rats with successful experimental anterior disc displacement $(n=6,4$, and 4 at 1,4 , and 8 weeks, respectively). There was no significant difference between the joints subjected to a sham operation and ADD (Mann-Whitney $U$ test, $P>0.05$ ), or among the follow-up time points (Friedman test, $P>0.05$ ).
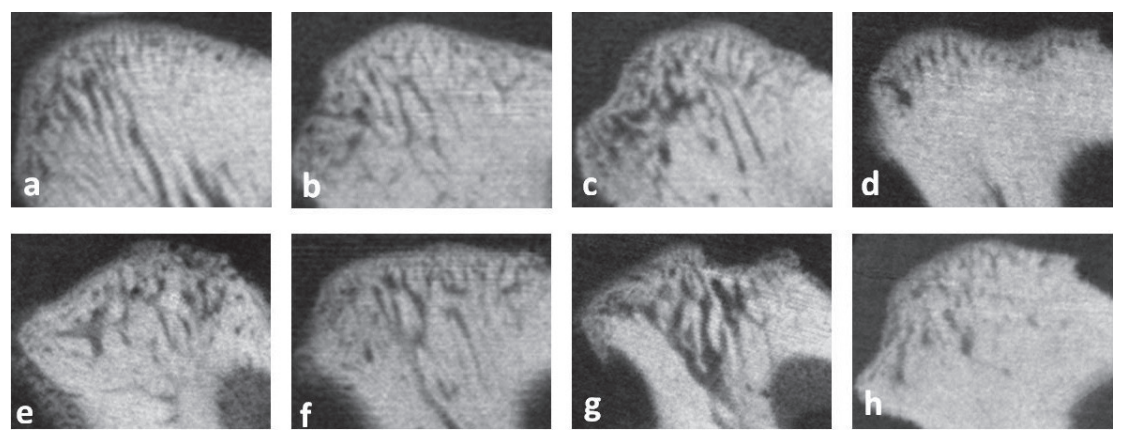

Fig. 4 Micro-CT scan assessment. A. normal condyles: a, control; b, sham operation; c, 4-week ADD. B. abnormal osseous changes: d, concavity control; e, erosion 1-week ADD; f, flattening 1-week ADD; g, concavity 1-week ADD; h, osteophyte 8-week ADD

Table 1 Condylar bony changes revealed by micro-CT

\begin{tabular}{|c|c|c|c|c|c|c|c|}
\hline Group & \multicolumn{2}{|c|}{$\begin{array}{c}\text { Follow-up time point } \\
\text { (number of rats) }\end{array}$} & Normal & Erosion & Concavity & Flattening & Osteophyte \\
\hline \multirow{2}{*}{ Control } & \multirow{2}{*}{$\begin{array}{c}0 \\
(n=4)\end{array}$} & $\mathrm{R}$ & 2 & 0 & 1 & 1 & 0 \\
\hline & & $\mathrm{L}$ & 3 & 0 & 0 & 0 & 0 \\
\hline \multirow{6}{*}{ Sham } & \multirow{2}{*}{$\begin{array}{l}1 \text { week } \\
(n=4)\end{array}$} & $\mathrm{R}$ & 3 & 0 & 0 & 0 & 1 \\
\hline & & $\mathrm{L}$ & 3 & 0 & 1 & 0 & 0 \\
\hline & \multirow{2}{*}{$\begin{array}{c}4 \text { weeks } \\
(n=4)\end{array}$} & $\mathrm{R}$ & 3 & 0 & 0 & 0 & 1 \\
\hline & & $\mathrm{L}$ & 4 & 0 & 0 & 0 & 0 \\
\hline & \multirow{2}{*}{$\begin{array}{c}8 \text { weeks } \\
(n=4)\end{array}$} & $\mathrm{R}$ & 3 & 0 & 1 & 0 & 0 \\
\hline & & $\mathrm{L}$ & 4 & 0 & 0 & 0 & 0 \\
\hline \multirow{6}{*}{ ADD } & \multirow{2}{*}{$\begin{array}{l}1 \text { week } \\
(n=6)\end{array}$} & $\mathrm{R}$ & 1 & 4 & 1 & 1 & 0 \\
\hline & & $\mathrm{L}$ & 6 & 0 & 0 & 1 & 0 \\
\hline & \multirow{2}{*}{$\begin{array}{r}4 \text { weeks } \\
(n=4) \\
\end{array}$} & $\mathrm{R}$ & 2 & 0 & 0 & 0 & 2 \\
\hline & & $\mathrm{L}$ & 4 & 0 & 0 & 0 & 0 \\
\hline & \multirow{2}{*}{$\begin{array}{r}8 \text { weeks } \\
(n=4)\end{array}$} & $\mathrm{R}$ & 2 & 0 & 0 & 1 & 1 \\
\hline & & $\mathrm{L}$ & 4 & 0 & 0 & 0 & 0 \\
\hline
\end{tabular}

$\mathrm{R}$, right condyle; L, left condyle. Data are shown as the number of condyles.

\section{Body weight changes}

There was a reduction in body weight in both groups during the first week of follow-up, but a steady weight gain was observed thereafter (Fig. 3). There was no significant difference in body weight between the sham and ADD groups at each of the follow-up time points during the experimental period (Student's $t$-test, $P>0.05$ ).

\section{Gross evaluation of ADD}

The operation site healed well in all rats, except for mild swelling a few days after surgery. During the first week, some rats in the sham and ADD groups showed unbalanced attrition of the incisors with the inclination higher to the left side; however, tooth abrasion gradually resolved.

Gross examination after sacrifice showed that in 14 of the 24 rats (six, four, and four rats sacrificed at 1,4 , and 8 weeks, respectively) in the ADD surgery group, the TMJ disc remained in an anteriorly displaced position, i.e. the posterior attachment was superior to the condyle. Unsuccessful cases were attributable to disc fragmentation $(n=3)$, disc perforation ( $n$ $=2$ ), and changing the fixation position on the zygomatic arch $(n=8)$, which caused suture loosening, resulting in the disc returning to its normal position.

\section{Micro-CT scan}

Table 1 details the micro-CT assessment related to osseous abnormalities. In the ADD group, condylar osseous changes showed that erosion or concavity signs tended to develop in experimental joints 1 week after surgery (Fig. 4). However, these signs were found to have disappeared on later follow-up scans, and cortical bone was obviously evident in 8-week ADD joints.

\section{Histological results}

Quantitative evaluation

As shown in Fig. 5, condylar cartilage thickness in the posterior region of 

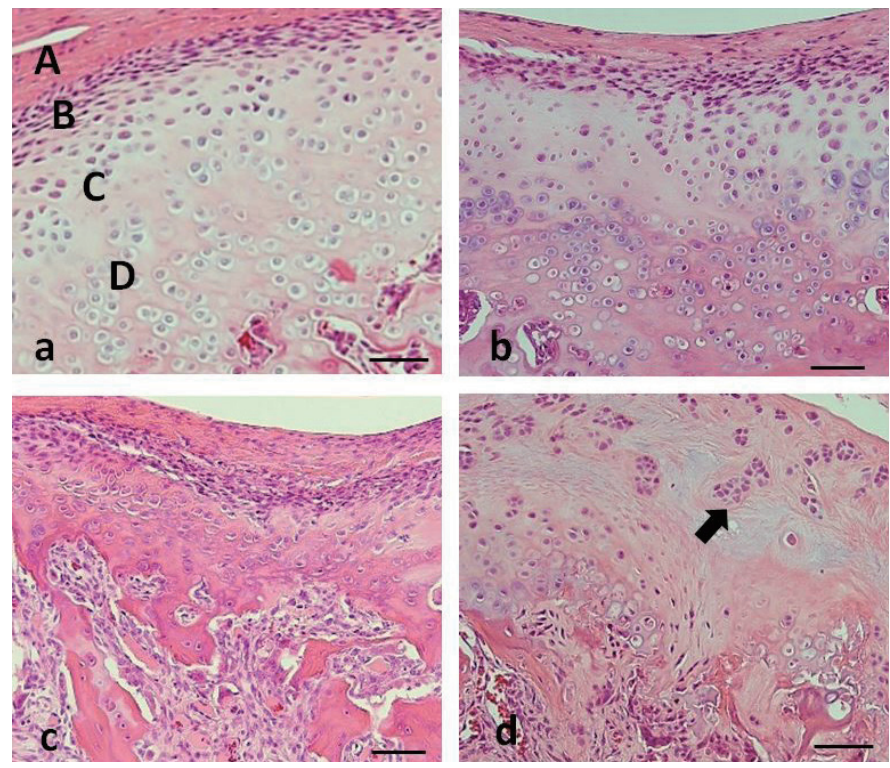

Fig. 6 Histological characteristics. a, control joint with four distinct layers: A, Fibrous; B, Proliferative; C, Chondroblastic; D, Hypertrophic. b, 1-week ADD showed loss of layer organization, with cartilage hyperplasia. c, 1-week ADD exhibited absence of specific layers. d, clusters of chondrocytes in the fibrous layer (arrow) and cellular atrophy (HE, hematoxylin and eosin; bar represents $50 \mu \mathrm{m})$

the condyle was decreased in the ADD groups at 4 and 8 weeks; however, there was no statistically significant difference between sham and ADD joints (Mann-Whitney $U$ test, $P>0.05$ ), or among the various follow-up time points (Friedman test, $P>0.05$ ).

\section{Qualitative evaluation}

Condylar cartilage: In four non-treated joints and three sham-treated joints, micro-CT images showed abnormal osseous changes, including flattening, concavity, and osteophytes (Table 1); however, histological examination demonstrated no notable difference from the other normal joints in terms of well-defined organized layers and cellular arrangement of the condylar cartilage. Therefore, there was no noticeable difference in histological characteristics between the non-treated and sham-treated joints, all of which were considered to constitute "control" preparations.

In control joints, articular cartilage is regionalized to four clear layers: fibrous, proliferative, chondroblastic, and hypertrophic layers.

In the anterior region of the condyle, in 1-week ADD joints, obvious histological changes were observed. Structural disarrangement was not uniform, and some unique characteristics were evident. In the same experimental condyle, cartilage hyperplasia and hypoplasia coexisted. Some areas showed a decrease in cartilage thickness, loss of layer organization or absence of specific layers (Fig. 6). Most of these alterations were also observed in the 4-week ADD joints to a lesser degree. However, the ADD joints in the 8-week group showed improvement as severe cellular atrophy and cartilage hyperplasia disappeared. In two joints, four well-organized layer structures appeared, even though the thickness had not yet normalized (Fig. 7).

The posterior region of the condyle showed less change than the anterior region; the layers remained intact. Hyperplasia was noteworthy, especially in 1-week ADD. However, only minor changes in cellular arrangement were observed in 4-week ADD joints. Furthermore, normal articular cartilage was seen in most of the 8-week ADD specimens.

Glenoid fossa: The articular surface of control joints was composed of a superficial fibrous layer and an underlying chondrocyte layer. The glenoid surface was altered with cell clusters and regional loss of cells in most 1-week and 4-week ADD joints. Erosion was seen in two 1-week joints. Eight-week ADD joints showed tissue rearrangement to a lesser degree in some scattered areas.

Disc: Control discs were double-concave and the central thinnest part was located superior to the posterocentral condyle. In ADD joints, the disc was elongated and/or deformed, the central zone being located anterior to the condyle.
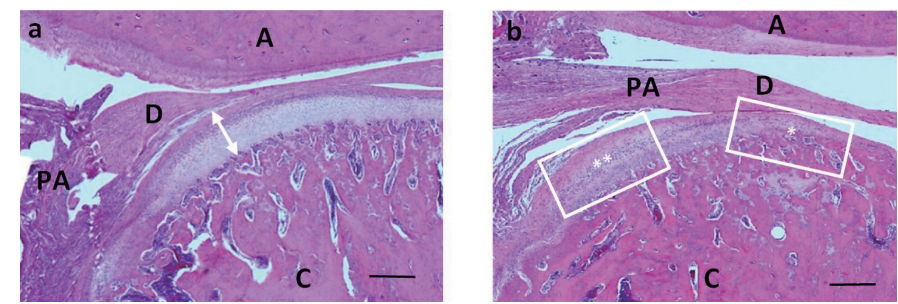

Fig. 7 a, control joint: A, articular fossa; C, condyle; D, disc; PA, posterior attachment. b, TMJ structure of an ADD joint, 8 weeks after surgery. The disc is displaced anteriorly, and the PA is superior to the condyle (HE; bar represents $300 \mu \mathrm{m}$ ). *, anterior condyle; **, Posterior condyle white double-ended arrow, area used to measure condylar cartilage thickness
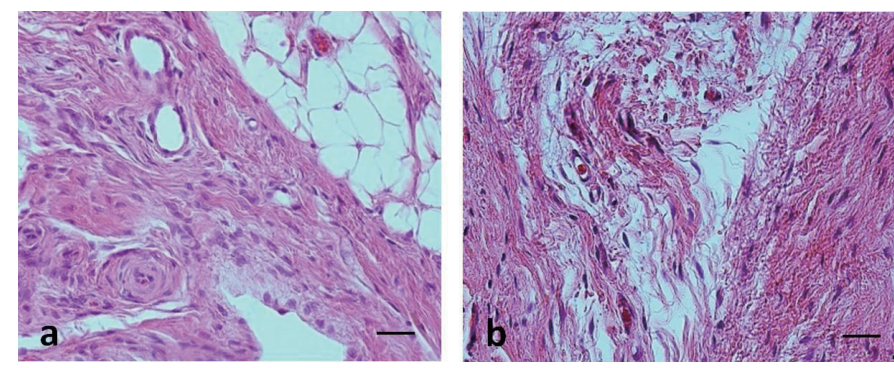

Fig. 8 Posterior attachment. a, control joint; b, 8-week ADD joint. The PA shows fibrosis of loose connective tissue with a reduced fatty component (HE; bar represents $50 \mu \mathrm{m}$ ).

Posterior attachment (PA): The PA of the TMJ in control rats was made up of loose fatty connective tissue. In ADD rats, there was an increase in fibroblasts and fibrous tissue, and a loss of adipose tissue, especially at 8 weeks (Fig. 8).

\section{Discussion}

The body weight decrease observed in both surgical groups during the first week may have been a consequence of postoperative pain, causing low food consumption, even though a soft diet was supplied. Such pain would have affected daily mastication and gnawing activity, thus contributing to unbalanced attrition of the incisors. However, after 1 week, rats returned to normal food consumption and mastication, accompanied by an increase in body weight and attenuation of incisal abrasion.

The presence of a high coronoid process in rats should be considered when deciding the position of the suture. Micro-CT evaluation was used to define the position of the hole in the zygomatic arch so that the coronoid process did not interfere with the suture and its direction could be made as anteriorly straight as possible.

Several surgical protocols for creating ADD models in rabbits have been reported $[18,21,22,35]$. However, these have differences, such as opening or not opening the joint, dissecting the discal attachments or keeping them intact, and the position at which the disc is sutured (anterior part of the disc or anterior extension of the disc). In the present study, a surgical method involving a direct approach to the joint was chosen because this helped to define the intra-articular structures and ensured correct displacement of the disc in relation to the condyle. Even though surgical opening of the joint was thought to affect joint stability, radiographical and histological evaluation revealed only minimal changes in the sham-treated joints. Since the anterior extension of the rats TMJ disc revealed thin and loose connective tissue, whereas the anterior part of the disc composed of fibrous tissue was much more compact, the latter was chosen as the suturing position.

In this study, the anterior region of the condyle was the most affected area following ADD, and the posterior part exhibited fewer changes. The TMJ is susceptible to mechanical stimuli. Remodeling process is capability of TMJ structures to adapt with changing forces applied on it. A sudden change in the position of the disc influenced the anterior condylar cartilage. Signs of osteoarthrosis were observed in most of the 1-week and 4-week ADD joints, but by 8 weeks after surgery, these signs were attenuated or resolved. A previous study using finite element models developed on the basis of MRI in two human subjects with or without ADD of the TMJ analyzed the stress distribution on condyles during prolonged clenching. 
This showed that the posterior area of the condylar articular surface in the ADD joint experienced higher stress, which might have caused the condylar cartilage to break down [7]. It is noteworthy that in this experiment, posterior condylar cartilage thickness decreased during the follow-up period, although not to a significant degree. Moreover, the posterior region of the condyle showed good qualitative recovery following disc displacement. The mandibular condyle has marked adaptive ability; in the absence of other contributing factors, such as prolonged clenching, ADD had a reduced influence on the posterior region of the condyle. However, these results were not consistent with previous ADD rabbit models. Many studies have described that osteoarthritic changes to the condyle persisted throughout follow-up periods of 6 weeks, 8 weeks and 24 weeks, respectively $[17,18,20]$. Interestingly, however, $\mathrm{Gu}$ et al. observed recovery after 8 weeks [21]. These studies indicate that differences in the surgical approach can affect the results.

In the present study, the severity of condylar change following surgery was not the same in all 1-week ADD rats. This may have been due to differences in individual reactions or pulling forces placed on the disc. Li et al. applied different forces to the TMJ discs of rabbits using elastic bands, and found a correlation between the stretching force and the severity of change in the condylar cartilage [22]. The present study used thread to induce ADD, and the position of the disc was restricted by discal attachments that remained intact. After displacing the disc, the success of ADD was evaluated by assessing the disc position. However, as the pulling force placed on the disc was difficult to control, differences among the rats may have been present. New controls should therefore be studied to ensure that similar forces are applied to the disc.

The PA of the normal TMJ is rich in blood vessels and nerves. In this study, the PA of ADD joints showed an increase in fibrous tissue. Fibrosis of the PA following ADD can originate from tensions in the posterior region and various loads from the condyle during function. This finding concurred with the results obtained from ADD-related cadaver or autopsy specimens [9,12], and also with results from a rabbit ADD model [21]. In patients with untreated ADD without reduction, MRI has revealed pseudo-disc sign, resulting from increased fibrous tissue in the PA [36,37]. During fibrosis in ADD joints, the PA might become less innervated, thus preventing transmission of noxious stimuli when the condyle functions on the PA instead of the disc. This might explain why many patients with ADD are pain-free. These may be considered as adaptive changes when the PA functions as a disc.

This study had some limitations, the main one being that the TMJ disc in $14(58.3 \%)$ of the 24 rats subjected to ADD remained in the anterior position at the time of sacrifice. Therefore, the present surgical technique needs to be modified to eliminate surgical failures, in order to make the ADD model more reproducible. Moreover, stretching force plays a crucial role in establishing any ADD model. Therefore, novel methods for ensuring that the same force is applied to the TMJ disc need to be considered.

Overall, this is the first reported study to have developed a model of temporomandibular ADD in rats by tracing the disc anteriorly via a nylon suture without dissection of the discal attachments. The success rate was $58.3 \%$. Various TMJ components responded in distinctive ways to ADD. Although osteoarthritic changes appeared in the initial postoperative period, they subsequently ameliorated. Within the limitations of this experiment, this model may be used to evaluate the progressive changes occurring after intra-articular derangement to further elucidate the etiology of osteoarthritis in the TMJ.

\section{Acknowledgments}

The authors are grateful to Dr. Kei Sakamoto for helpful discussions regarding the histological findings, Ms. Kiyoko Nagumo for valuable technical assistance with laboratory preparation, and Drs Liang Shanshan and Kay Thwe Ye Min Soe for assistance with surgery.

\section{Conflict of interest}

The authors have no conflict of interest, financial or otherwise, to declare.

\section{References}

1. Greene CS (2010) Managing the care of patients with temporomandibular disorders: a new guideline for care. J Am Dent Assoc 141, 1086-1088.
2. Ribeiro RF, Tallents RH, Katzberg RW, Murphy WC, Moss ME, Magalhaes AC et al. (1997) The prevalence of disc displacement in symptomatic and asymptomatic volunteers aged 6 to 25 years. J Orofac Pain 11, 37-47.

3. Larheim TA, Westesson P, Sano T (2001) Temporomandibular joint disk displacement: comparison in asymptomatic volunteers and patients. Radiology 218, 428-432.

4. Naeije M, Te Veldhuis AH, Te Veldhuis EC, Visscher CM, Lobbezoo F (2013) Disc displacement within the human temporomandibular joint: a systematic review of a 'noisy annoyance'. J Oral Rehabil 40, 139-158.

5. Stegenga B (2001) Osteoarthritis of the temporomandibular joint organ and its relationship to disc displacement. J Orofac Pain 15, 193-205.

6. Tanaka E, Hirose M, Koolstra JH, Van Eijden TM, Iwabuchi Y, Fujita R et al. (2008) Modeling of the effect of friction in the temporomandibular joint on displacement of its disc during prolonged clenching. J Oral Maxillofac Surg 66, 462-468.

7. Nishio C, Tanimoto K, Hirose M, Horiuchi S, Kuroda S, Tanne K et al. (2009) Stress analysis in the mandibular condyle during prolonged clenching: a theoretical approach with the finite element method. Proc Inst Mech Eng H 223, 739-748.

8. Aoun M, Mesnard M, Monède-Hocquard L, Ramos A (2014) Stress analysis of temporomandibular joint disc during maintained clenching using a viscohyperelastic finite element model. J Oral Maxillofac Surg 72, 1070-1077.

9. Luder HU (1993) Articular degeneration and remodeling in human temporomandibular joints with normal and abnormal disc position. J Orofac Pain 7, 391-402.

10. Pereira Junior FJ, Lundh H, Westesson PL (1996) Age-related changes of the retrodiscal tissues in the temporomandibular joint. J Oral Maxillofac Surg 54, 55-61.

11. Westesson PL, Rohlin M (1984) Internal derangement related to osteoarthrosis in temporomandibular joint autopsy specimens. Oral Surg Oral Med Oral Pathol 57, 17-22.

12. Kurita K, Westesson PL, Sternby NH, Eriksson L, Carlsson LE, Lundh H et al. (1989) Histologic features of the temporomandibular joint disk and posterior disk attachment: comparison of symptom-free persons with normally positioned disks and patients with internal derangement. Oral Surg Oral Med Oral Pathol 67, 635-643.

13. Pereira FJ, Lundh H, Eriksson L, Westesson PL (1996) Microscopic changes in the retrodiscal tissues of painful temporomandibular joints. J Oral Maxillofac Surg 54, 461-468.

14. Herring SW (2003) TMJ anatomy and animal models. J Musculoskelet Neuronal Interact 3 , 391-395.

15. Porto GG, Vasconcelos BC, Andrade ES, Silva-Junior VA (2010) Comparison between human and rat TMJ: anatomic and histopathologic features. Acta Cir Bras 25, 290-293.

16. Tomasello G, Sorce A, Mazzola M, Barone R, Lo Piccolo C, Farina F et al. (2016) Comparative analysis of the structure of temporomandibular joint in human and rabbit. Acta Biomed 87, 282-285.

17. Ali AM, Sharawy MM (1994) Histopathological changes in rabbit craniomandibular joint associated with experimentally induced anterior disk displacement (ADD). J Oral Pathol Med 23, 364-374.

18. Mills DK, Daniel JC, Herzog S, Scapino RP (1994) An animal model for studying mechanisms in human temporomandibular joint disc derangement. J Oral Maxillofac Surg 52, 1279-1292.

19. Kubota Y, Takatsuka S, Nakagawa K, Yamamoto E (2001) A model for temporomandibular joint disc repositioning surgery. J Oral Maxillofac Surg 59, 1443-1451.

20. Berteretche MV, Foucart JM, Meunier A, Carpentier P (2001) Histologic changes associated with experimental partial anterior disc displacement in the rabbit temporomandibular joint. J Orofac Pain 15, 306-319.

21. Gu Z, Zhou Y, Zhang Y, Zhao S, Liu J, Hu J (2006) An animal model for inducing anterior disc displacement of the temporomandibular joint. J Orofac Pain 20, 166-173.

22. Li H, Cai X, Wang S, Yang C, Song H, Huang L (2014) Disc positions and condylar changes induced by different stretching forces in the model for anterior disc displacement of temporomandibular joint. J Craniofac Surg 25, 2112-2116.

23. Hinton RJ (1992) Alterations in rat condylar cartilage following discectomy. J Dent Res 71, 1292-1297.

24. Hinton RJ, Stinson JL (1997) Effect of postoperative diet on condylar cartilage response to discectomy. J Oral Maxillofac Surg 55, 1259-1264.

25. Martini DT, De Campos Boldrini S, De Vasconcellos Fontes RB, Liberti EA (2006) Ultrastructural study of the temporomandibular joint after unilateral meniscectomy in Wistar rats. J Oral Rehabil 33, 722-728.

26. Nicoll SB, Hee CK, Davis MB, Winkelstein BA (2010) A rat model of temporomandibular joint pain with histopathologic modifications. J Orofac Pain 24, 298-304.

27. Chisnoiu AM, Chisnoiu R, Moldovan M, Lascu LM, Picos AM (2016) Etiological factors associated with temporomandibular joint disorder - study on animal model. Rom J Morphol Embryol 57, 185-189.

28. Ardran GM, Kemp FH, Ride WDL (1958) A radiographic analysis of mastication and swallowing in the domestic rabbit: oryctolagus cuniculus (L). J Zool 130, 257-274.

29. Hiiemäe KM, Ardran GM (1968) A cinefluorographic study of mandibular movement during feeding in the rat (Rattus norvegicus). J Zool 154, 139-154

30. Risnes S (1973) Mandibular movement capacity in the rat. Scand J Dent Res 81, 170-173.

31. Bouvier M (1988) Effects of age on the ability of the rat temporomandibular joint to respond to changing functional demands. J Dent Res 67, 1206-1212.

32. Sengupta P (2013) The laboratory rat: relating its age with human's. Int J of Prev Med 4 , 624-630.

33. Kameoka S, Kuroki Y, Honda K, Kijima N, Matsumoto K, Asano M et al. (2009) Diagnostic accuracy of microcomputed tomography for osseous abnormalities in the rat temporomandibular joint condyle. Dentomaxillofac Radiol 38, 465-469.

34. Schneider CA, Rasband WS, Eliceiri KW (2012) NIH Image to ImageJ: 25 years of image analysis. Nat Methods 9, 671-675.

35. Ali AM, Sharawy M, O’Dell NL, Al-Behery G (1993) Morphological alterations in the elastic fibers of the rabbit craniomandibular joint following experimentally induced anterior disk displacement. Acta Anat (Basel) 147, 159-167.

36. Katzberg RW, Bessette RW, Tallents RH, Plewes DB, Manzione JV, Schenck JF et al. (1986) Normal and abnormal temporomandibular joint: MR imaging with surface coil. Radiology 158, 183-189.

37. Zhuo Z, Cai XY (2016) Radiological follow-up results of untreated anterior disc displacement without reduction in adults. Int J Oral Maxillofac Surg 45, 308-312. 